\section{Caries control in health service practice}

\author{
J. Page, ${ }^{1}$ J. A. Weld ${ }^{2}$ and E. A. M. Kidd ${ }^{3}$
}

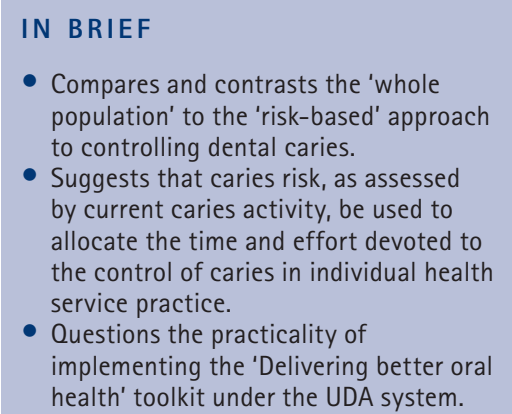

- Compares and contrasts the 'whole population' to the 'risk-based' approach to controlling dental caries.

Suggests that caries risk, as assessed by current caries activity, be used to allocate the time and effort devoted to the control of caries in individual health service practice.

implementing the 'Delivering better ora health' toolkit under the UDA system.

It is suggested that it makes sense for dentists providing care for individual patients to take account of caries risk (as assessed by presentation of active, non-cavitated lesions) when deciding how to allocate time and effort of themselves and their staff. However, there is a question as to how realistic it is to ask the dental team to provide a full diagnostic assessment and all the preventive treatment required for a patient for the payment provided by 1 UDA. It is to be hoped that one or more of the Steele pilots will come up with a practical solution for controlling caries in NHS practice.

In September 2007 the Department of Health published and circulated Delivering better oral health: an evidence based toolkit for prevention. This was updated in 2009, ${ }^{1}$ and again distributed to every NHS practice in England. The Toolkit provides evidence-based guidance for preventive care in practice. For caries control it stresses the importance of fluoride toothpaste and surgery application of fluoride varnish. It advocates a whole population approach to prevention of dental caries and this was strongly supported in a $B D J$ editorial by Tickle and Milsom. ${ }^{2}$ We fully support their contention that prevention should be taken seriously. However, it is apparent that, despite the best efforts of many dentists, operative treatment has usually taken priority over the preventive treatment of dental caries. It appears neither the Department of Health nor the General Dental Services Committee of the BDA has developed a system of payment to encourage effective preventive rather than operative treatment in general practice. In our opinion the new contract introduced in 2006 which was meant to facilitate access

${ }^{1 *}$ Specialist in Paediatric Dentistry, ${ }^{2}$ Clinical Director, Seagull Apartment, 13A Broad Street, Portsmouth, P01 2JD; ${ }^{3}$ Emerita Professor of Cariology, King's College London Hillcroft, 57 Langley Avenue, Surbiton, Surrey, KT6 60R.

${ }^{*}$ Correspondence to: Dr Jim Page

Email:jimpage@me.com

\section{Refereed Paper}

Accepted 9 April 2010

DOI: $10.1038 /$ sj.bdj.2010.447

${ }^{\circledR}$ British Dental Journal 2010; 208: 449-450 to dental care and promote preventive treatments has done neither. Indeed, we believe it may be even more difficult for the practitioner to concentrate on prevention in the post 2006 contract.

\section{Whole population or risk based approach}

It is widely accepted that the major improvements in human health over the last 150 years have been due to a 'whole population' approach rather than a 'risk based' approach as emphasised by Geoffrey Rose in 1985. ${ }^{3}$ This 'whole population' approach is appropriate to the prevention of dental disease and is the only way to reduce the burden of disease and the cost of dental care. ${ }^{4}$ But Rose gave a balanced view of the advantages and disadvantages of both the 'high-risk strategy' and the 'population strategy' and in conclusion stated "The "high-risk" strategy of prevention is an interim expedient, needed in order to protect susceptible individuals, but only for so long as the underlying causes of incidence remain unknown or uncontrollable; if causes can be removed, susceptibility ceases to matter. Realistically, many diseases will long continue to call for both approaches, and fortunately competition between them is usually unnecessary. ${ }^{3} \mathrm{We}$ know the causes of dental caries but at present are not able completely to control the disease either for the population as a whole or for every individual patient.

We fully support the concentration of effort in the Toolkit on the use of an appropriate dose of fluoride toothpaste. Plaque is the cause of caries and its disturbance twice daily with a fluoride containing dentifrice should be the bedrock of caries control in all patients: the whole population approach. However, while accepting the application of fluoride varnish is an effective way of reducing the incidence of caries, the suggestion it should be applied to all children, 'the whole population approach', is surprising in view of cost, even if applied by dental nurses. The Cochrane systematic review ${ }^{5}$ did not provide information on the cost effectiveness of the procedure but a further systematic review ${ }^{6,7}$ looked at cost and concluded that 'Any protocol on the application of fluoride varnish should be based on risk assessment. The best indicator of risk of caries is previous or current caries experience'. It would seem to us a 'whole population' approach to prevention is appropriate for public dental health initiatives ${ }^{4}$ but it still makes sense for dentists providing care for individual patients to take account of caries risk (as assessed by presentation of active, non-cavitated lesions) when deciding how to allocate time and effort of themselves and their staff. As Rose intimated, both approaches are still appropriate for dental caries.

\section{The importance of diagnosis of the early lesion and diagnosis of caries risk}

We would disagree with Tickle and Milsom that paying dental nurses to apply fluoride 
varnish may be preferable to paying dentists to 'hunt for early lesions'. We suggest it is vital that dentists make efforts to diagnose the early lesion so the skills of the dental team can be put to best use.

Tickle and Milsom contend it is not possible to identify children who will develop caries quoting a paper ${ }^{8}$ looking at risk factors for caries in children under six years. This paper did not consider existing or previous caries as a risk factor. However, this is known to be the most accurate predictor of risk. ${ }^{9,11}$ Hausen et al. ${ }^{10}$ have shown a significant reduction in caries increment when targeting caries preventive measures at children with active non-cavitated lesions. In addition a 'risk based' approach is advocated by NICE ${ }^{11}$ to determine recall intervals. Thus, perhaps risk prediction should not be dismissed.

Tickle and Milsom state that their work ${ }^{12}$ suggests that whatever age caries starts in young children, it progresses at the same rapid rate. This view surprises us but perhaps derives from the fact that their study looked at the progress of dental caries as 'no cavity - to - cavity'. Non-cavitated lesions were not counted and virtually no radiographs were taken. It is likely that if you see no cavity at one visit and a cavity at a subsequent visit, you will define the development as 'rapid'. However, in most children caries progresses slowly and this will be appreciated by all clinicians who 'hunt for early lesions' and then put in place more intensive preventive measures for these patients who have proved themselves to be at higher risk. The reference in their editorial to Axelsson's work implies that he uses a 'whole population' approach. However, reference to the paper ${ }^{13}$ indicates that he uses in fact a 'risk based' approach to the prevention of caries in his population of patients.

While it is acknowledged that caries risk prediction is far from perfect, we cannot believe that it is right in general dental practice to spend the same amount of time and effort on prevention for every patient regardless of their risk of future disease as suggested in their editorial. We totally agree it is the place of the profession and the Department of Health to push for effective population based preventive measures, such as campaigns on oral hygiene with fluoride containing toothpaste and diet (especially where there is a common risk such as obesity).

Perhaps school based fluoride varnish application is also appropriate although a recent cluster randomised controlled trial failed to show a reduction in caries. ${ }^{14}$ One of the reasons for this lack of effect appeared to be that the parents of the children at high risk did not consent to treatment and the children that were consented had low caries levels to start with. So further evidence is required to assess the cost-effectiveness of universal fluoride varnish application.

\section{The practicalities of implementing the Toolkit under the UDA system of payment}

Under the UDA system, there are three bands of payment and Band 1 of the contract is described as: Diagnosis and treatment planning and maintenance. This links preventive treatments with diagnosis within a single band. There is no uniform value of UDA. The average value is said to be $£ 24.38$ which is the gross figure out of which all practice expenses have to be paid (figures produced by the National Association of Specialist Dental Accountants ${ }^{15}$ )and since this is an average some will be paid more and some less by their PCT. So how much time do these values amount to in surgery time? We would suggest the £24 might represent 15 minutes which must include:

- Getting the patient in and out of the chair

- Implementing appropriate cross infection control measures

- Greeting and communicating with the patient

- Writing up the patient's record.

What else must the dental team include in this time? We suggest the following:

- The history and clinical examination according to NICE guidelines ${ }^{11}$

- Any necessary radiographs

- Formulate the treatment plan, explain it to the patient and gain their consent

- All preventive treatments - as advised in the Toolkit:

o oral hygiene instruction

o dietary advice if appropriate

- fissure sealing if appropriate

○ fluoride varnish application for all children and young adults

- Scaling and polishing if required.

To suggest this could be done for $£ 24$, in 15 minutes, is in our opinion a deception.

Who is being deceived? We suggest it is the patient, to such an extent that we consider the GDC, whose role is to protect the patient, should be interested.

It means the whole UDA system is founded on something that is unattainable and therefore, we consider, unethical.

We would suggest that even Tickle and Milsom, with their use of less sensitive caries diagnostic criteria, could not achieve what is required by the contract for the payment provided by one UDA.

1. Department of Health and British Society for the Study of Community Dentistry. Delivering better oral health: an evidence-based toolkit for prevention, 2nd ed. Gateway ref 8504. 2009.

2. Tickle M, Milsom K. The whole population approach to caries prevention in general dental practice. $\mathrm{Br}$ Dent J 2008; 205: 521.

3. Rose G. Sick individuals and sick populations. Int J Epidemiol 1985; 14: 32-38

4. Batchelor PA, Sheiham A. The distribution of burden of dental caries in schoolchildren: a critique of the high-risk caries prevention strategy for populations. BMC Oral Health 2006; 6: 3.

5. Marinho V C C, Higgins J P T, Logan S, Sheiham A. Fluoride varnishes for preventing dental caries in children and adolescents. Cochrane Database Syst Rev 2002; CD002279.

6. Azarpazhooh A, Main P A. Fluoride varnish in the prevention of dental caries in children and adolescents: a systematic review. J Can Dent Assoc 2008; 74: 73-79.

7. Quinonez R B, Stearns S C, Talekar B S, Rozier R G, Downs $S$ M. Simulating cost-effectiveness of fluoride varnish during well-child visits for Medicaidenrolled children. Arch Pediatr Adolesc Med 2006; 160: 164-170.

8. Harris R, Nicoll A D, Adair P M, Pine C M. Risk factors for dental caries in young children: a systematic review of the literature. Community Dent Health 2004; 21(Suppl): 71-85.

9. Hausen H. Caries prediction. In Fejerskov O, Kidd E (eds). Dental caries - the disease and its clinical management, 2nd ed. pp 527-542. Blackwell Munksgaard, 2008.

10. Hausen H, Seppa L, Poutanen R, Niinimaa A et al. Noninvasive control of dental caries in children with active initial lesions. A randomized clinical trial. Caries Res 2007; 41: 384-391.

11. NICE guideline. Dental recall - recall interval between routine dental examinations. http://www.nice.org.uk/guidance/index. jsp?action $=$ downloadEto $=29488$

12. Milsom K M, Blinkhorn A S, Tickle M. The incidence of dental caries in the primary molar teeth of young children receiving National Health Service funded dental care in practices in the North West of England. Br Dent J 2008; 205: E14.

13. Axelsson P. The effect of a needs-related caries preventive program in children and young adultsresults after 20 years. BMC Oral Health 2006; 6(Suppl 1): S7.

14. Hardman M C, Davies G M, Duxbury J T, Davies R M A cluster randomised controlled trial to evaluate the effectiveness of fluoride varnish as a public health measure to reduce caries in children. Caries Res 2007; 41: 371-376.

15. Watson M. Private up, NHS down. Dent Pract 2009; 47: 1. 